\title{
Optical model for multilayer glazing systems: application to laminated glass and photovoltaic modules.
}

\author{
TOMÁS BAENAS ${ }^{*}$, MAIDER MACHADO \\ ${ }^{1}$ Applied Mathematics Department. University of Alicante. Carretera de S. Vicente del Raspeig s/n. 03690 \\ S. Vicente del Raspeig. Alicante (Spain). tomas.baenas@ua.es \\ 2Energy and Environment Division. Tecnalia Research and Innovation. Paseo Mikeletegi, 2. 20009 San \\ Sebastián (Spain). maider.machado@tecnalia.com
}

This article provides theoretical developments broadening the scope of previous optical simulation models for multilayer glazing systems. The applicability of existing models will be extended through additional characterization of the multilayer optical components from global spectrophotometric (UV-Vis-NIR) measurements. A more complete interlayer film characterization, including reflectivity in the film-glass interface, will be provided. Singular solutions of the related equation systems will be derived for situations involving components with very low or null transmissivity. As a contribution to the fundamentals of the formalism, the condition relating the symmetry of the transmittance of the system with the symmetry of the transmissivity of its optical components will be studied. Finally, with the extension for the calculation of energy fluxes through the components of a multilayer system, analytical expressions for the components absorptivity will be derived. These results are particularly useful to quantify differences in energy absorption of the constituents of a laminated glass, as a tool to define, from the glazing design phase, the thermal and mechanical processing needed for each glazing component. Additionally, the model provides a procedure for the calculation of the absorptivity of encapsulated photovoltaic cells, which is directly related to cell efficiency in each particular configuration.

Key words: energy simulation, optical model, matrix method, laminated glass, photovoltaic module.

\footnotetext{
* Corresponding author
} 


\section{INTRODUCTION}

The current European regulatory framework (EN 410:2011, EN 673:2011, EN 13363:2003, etc.), and international regulations (ISO 9050:2003, ISO 15099:2003. etc.), for the determination of the optical and thermal performance of glazing systems are based on the provision of closed analytical expressions for the related short-wave energy magnitudes (transmittance, reflectance, absorptance), in terms of the energy performance of the system components (transmissivity, reflectivity, absorptivity). The latter are measurable from elementary configurations, while basic thermal constants are fixed by the standards.

Within this framework, the optical modeling of glazing systems avoids the setting of equations requiring numerical solution methods, while keeping a sufficient accuracy guaranteeing the thermal study of the systems. The propagation of solar radiation through complex optical systems with planoparallel optical interfaces has been previously studied by Baenas and Machado (2009), with a basis on the classical transfer matrix methods (Harbecke 1986, Maestre 2000, Pfrommer et al. 1995, Van Nijnatten 1994 , i.a.). The physical description is based on irradiance - incoming energy flux - and radiosity - outcoming energy flux - under the approximation of incoherent superposition of radiation waves, due to the fact that radiation wavelengths are negligible with respect to thickness in the case of glass substrates. Light diffusing elements or shading elements are not included. The formalism does not include angledependent optical properties (Furler 1991, Maestre et al. 2006, Rubin 1982, Rubin et al. 1998, Van Nijnatten 2001). These restrictions are aligned with the European standard for energy characterization of glass for building, EN 410:2011. Within this framework, the optical system is formed by glass panes, polymer films, selective coatings and gas spaces without optical function. This kind of system is symmetric regarding transmissivity, and can be characterized, to a good approximation, by means of UVVis-NIR spectrophotometry for normal incidence.

The optical model presented herein is based on the separation of a real optical glazing system in optical components (interfaces, glass substrates, films and coatings, mainly), which are defined through a transfer matrix connecting energy fluxes on each side of the component. Transfer matrix methods have been largely studied in the literature from different approaches (Centurioni 2005, Harbecke 1986, Katsidis and Siapkas 2002, Maestre 2000, Mitsas and Siapkas 1995, Pfrommer et al. 1995, Van Nijnatten 1994, i.a.). These methods have their roots in the classical study of characteristic matrices (dependent on Fresnel coefficients for interference systems or coherent case) which connect the electromagnetic field components at an interface (Abelès 1950, Born and Wolf 1959, Heavens 1960, etc.) and the application of the method to thin films (Epstein 1952, Francombe and Hoffman 1971, Herpin 1947, Thelen 1989, etc.). The reformulation of these matrices in terms of coefficients relating energy magnitudes (through, e.g., the Poynting vector) allows the definition of transfer matrices as proposed herein.

This paper provides a method for the systematic formulation of the equations for the determination of the energy coefficients of a glazing system and the experimental characterization of its components (Section 2). These operations and those related with the calculation of energy fluxes and absorptivities may be performed by means of an analytical mathematical formalism based on matrix calculations (Section 4). A review on the main hypothesis for the optical and energy modeling of multilayer systems will be presented, in particular regarding the null reflectivity of glass-film interfaces in a 
laminated glass (Section 3.2). The extension of the formalism for the characterization of opaque components (Section 3.1) and the calculation of absorptivities will allow the study of the energy distribution within a conventional laminated glass (Section 5.1) and a laminated photovoltaic module with glass superstrate (Section 5.2). Both cases illustrate the procedure to reduce the mathematical complexity of the problem during the modeling phase and to facilitate the formulation of closed analytical expressions.

\section{SUMMARY OF THE MODEL (OPERATIONAL ASPECTS)}

Despite the variety of existing definitions for transfer matrices, all the formalisms are equivalent and leading to the same algebraic relations between spectral reflectivities $(\rho)$ and transmissivities $(\tau)$ of the optical system components. Within the most frequent definition (Harbecke 1986, Pfrommer 1995) the transfer matrix $(M)$ relates external $(f$ front) and internal $\left(b\right.$ - back) irradiance $(I)$ and radiosity $(J)$ as $\left(I_{f}, J_{f}\right)^{t}=M\left(J_{b}, I_{b}\right)^{t}$, where superscript $t$ indicates the transpose matrix. Solar irradiance incidence defines the external side of the glazing. Within this convention, consecutive interfaces are connected by a transfer matrix product, where the radiosity of an interface is taken as irradiance for the next surface, and vice versa. The introduction of components with only energy absorption functionality requires a different type of matrix, given that the absorption process must not alter the order between incoming and outgoing fluxes.

In this paper, as in Baenas and Machado (2009), the definition convention given by Maestre (2000) has been followed, so that the relation between irradiance and radiosity was of the type $\left(I_{f}, J_{f}\right)^{t}=M\left(I_{b}, J_{b}\right)^{t}$. This allows the use of the same kind of matrix for interfaces and elements whose only function is the incoherent attenuation of radiation intensity. In return, an additional link matrix $(U)$ is needed for the irradiance-radiosity conversion at the junction between successive interfaces. From this definition, the explicit expression for transfer matrix is, in the general asymmetric case $\tau_{f} \neq \tau_{b}$,

$$
M=\frac{1}{\tau_{f}}\left(\begin{array}{cc}
-\rho_{b} & 1 \\
\tau_{f} \tau_{b}-\rho_{f} \rho_{b} & \rho_{f}
\end{array}\right), \quad U=\left(\begin{array}{ll}
0 & 1 \\
1 & 0
\end{array}\right)
$$

It should be noted that $M$ matrix is not defined in the case of null transmissivity, $\tau_{f}=0$, and that in the symmetric case it is a unimodular matrix, with $\operatorname{det}(M)=\operatorname{det}(U)=-1$. In spite of the apparent equivalence between both definitions, the last one simplifies the formal study of part of the contents of the model, as it allows an equivalent treatment of the transmissivity of each element, avoiding the need to define its function within the system (this is the case of absorptive selective coatings, polymer films and substrates). 


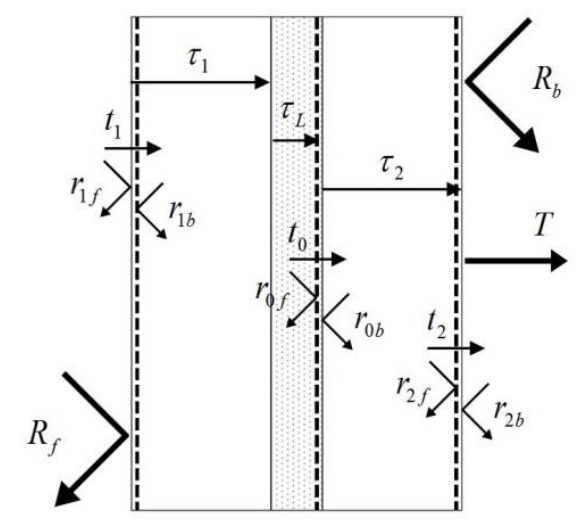

Fig. 1: Schematics and notation for a laminated glazing system.

One of the objectives of the method proposed by Baenas and Machado (2009) was to simplify the modelling of a glazing system, by developing a catalogue of the optical components (from their associated transfer matrices) which are closest to real system components. In this way, the reduction of a glazing into a sequence of optical interfaces and their characterization process, which would require an advanced knowledge of the subject, is avoided. The schematization of the system is then straightforward and has a direct translation into the setting up of the corresponding equation system. As an example, a monolithic glass with an internal coating is represented by "[S]|", as the junction of the following optical components: air-glass interface "[", glass substrate " $\mathrm{S}$ " and glass-air coating "]|".

Each i-th component has an associated $M_{c, i}$ matrix from the catalogue ( $c$ represents the type of component following Table 1). This matrix describes the function of the component within the system and includes the magnitudes which characterize it (transmissivity, reflectivities and the relations among them). In order to obtain these magnitudes from transmittance and reflectance spectrophotometric measurements of the glazing system, basic configurations must be considered (elementary glazing systems detailed below).

The transfer matrix of the glazing system, $M_{1, n}, n$ being the number of components, is obtained as the ordered product (front to back) of the transfer matrix of each optical component of the catalogue. This matrix relates the incoming and outgoing irradiance and radiosity pairs of the global optical system as,

$$
\left(\begin{array}{l}
I_{1, f} \\
J_{1, f}
\end{array}\right)=M_{1, n}\left(\begin{array}{l}
I_{n, b} \\
J_{n, b}
\end{array}\right), \quad M_{1, n}=M_{c, 1} M_{c, 2} \ldots M_{c, n}
$$

Where the numerical sub-index is correlative for the numbering of components. The symmetrical transmittance $\left(T=T_{f}=T_{b}\right)$ and reflectances $\left(R_{f}, R_{b}\right)$ of the optical system are obtained, by definition, from an unitary incident irradiance:

$$
\left(\begin{array}{c}
1 \\
R_{f}
\end{array}\right)=M_{1, n}\left(\begin{array}{c}
0 \\
T
\end{array}\right), \quad\left(\begin{array}{c}
0 \\
T
\end{array}\right)=M_{1, n}\left(\begin{array}{c}
1 \\
R_{b}
\end{array}\right)
$$

Expressions (3) can be rewritten as the transfer matrix of an equivalent optical interface for the glazing system, defined from (1), i.e., with $T \neq 0$, 


$$
M_{1, n}=\left(\begin{array}{ll}
m_{11} & m_{12} \\
m_{21} & m_{22}
\end{array}\right)=\frac{1}{T}\left(\begin{array}{cc}
-R_{b} & 1 \\
T^{2}-R_{f} R_{b} & R_{f}
\end{array}\right) .
$$

Therefore, from $m_{i j}$ matrix elements, the relations between the glazing energy coefficients $\left(T, R_{f}, R_{b}\right)$ and the coefficients describing the glazing components $\left(\tau_{i}, \rho_{i f}\right.$, $\left.\rho_{i b}\right)$ are given by $T=m_{12}^{-1}, R_{f}=m_{22} m_{12}^{-1}, R_{b}=-m_{11} m_{12}^{-1}$.

Table 1: Catalogue of optical components and transfer matrices.

\begin{tabular}{|c|c|c|c|}
\hline Component & Symbol & Transfer matrix & Matrix structure \\
\hline Glazing & & $\left(\begin{array}{cc}-\frac{w_{v}}{T} & \bar{T} \\
T-\frac{R_{f} R_{b}}{T} & \frac{R_{f}}{T}\end{array}\right)$ & $\begin{array}{l}T: \text { Transmittance } \\
R_{f}, R_{b}: \text { Reflectance front, back }\end{array}$ \\
\hline Air-glass interface & {[} & $\left(\begin{array}{cc}\frac{1}{1-r_{s}} & -\frac{r_{s}}{1-r_{s}} \\
\frac{r_{s}}{1-r_{s}} & 1-r_{s}-\frac{r_{s}^{2}}{1-r_{s}}\end{array}\right)$ & $\begin{array}{l}M_{\mathrm{I}}=M U \\
r_{s}: \text { Glass substrate reflectivity }\end{array}$ \\
\hline Glass-air interface & ] & $\left(\begin{array}{cc}1-r_{s}-\frac{r_{s}^{2}}{1-r_{s}} & \frac{r_{s}}{1-r_{s}} \\
-\frac{r_{s}}{1-r_{s}} & \frac{1}{1-r_{s}}\end{array}\right)$ & $\begin{array}{l}M_{]}=U M \\
r_{s}: \text { Glass substrate reflectivity }\end{array}$ \\
\hline Glass substrate & $S$ & $\left(\begin{array}{cc}0 & \frac{1}{\tau_{s}} \\
\tau_{s} & 0\end{array}\right)$ & $\begin{array}{l}M_{S}=M \\
\tau_{s}: \text { Glass substrate transmissivity }\end{array}$ \\
\hline Air-glass coating & |[ & $\left(\begin{array}{cc}\frac{1}{t_{0}} & -\frac{r_{0 b}}{t_{0}}\end{array}\right)$ & $\begin{array}{l}M_{\mid \mathrm{I}}=M_{\mathrm{C}}=M U \\
t_{0 f}: \text { Coating transmissivity }\end{array}$ \\
\hline Film-glass coating & ( & $\left(\begin{array}{ll}\frac{r_{0 f}}{t_{0}} & t_{0}-\frac{r_{0 f} r_{0 b}}{t_{0}}\end{array}\right)$ & $r_{0 f}, r_{0 b}$ : Coating reflectivity front, back \\
\hline Glass-air coating & ]1 & $\left(t_{0}-\frac{r_{0 f} r_{0 b}}{t_{0}} \frac{r_{0 f}}{t_{0}}\right)$ & $\begin{array}{l}M_{||}=M_{\jmath}=U M \\
t .\end{array}$ \\
\hline Glass-film coating & ) & $\left(\begin{array}{ll}-\frac{r_{0 b}}{t_{0}} & \frac{1}{t_{0}}\end{array}\right)$ & $\begin{array}{l}u_{0 f}: \text { oatuing transmissivity } \\
r_{0 f}, r_{0 b} \text { : Coating reflectivity front, back }\end{array}$ \\
\hline Film (no reflectivity) & ]+[ & $\left(\begin{array}{cc}0 & \tau_{L}^{*} \\
1 & 0 \\
\tau_{L}^{*} & 0\end{array}\right)$ & $\begin{array}{l}M_{1+[}=U M U \\
\tau_{L}^{*}: \text { Film transmissivity }\end{array}$ \\
\hline Film (with reflectivity) & )+( & $\left(\begin{array}{cc}\frac{\rho_{L}}{\tau_{L}} & \tau_{L}-\frac{\rho_{L}^{2}}{\tau_{L}} \\
\frac{1}{\tau_{L}} & -\frac{\rho_{L}}{\tau_{L}}\end{array}\right)$ & $\begin{array}{l}M_{\mathrm{T}+(}=U M U \\
\tau_{L}: \text { Film transmissivity } \\
\rho_{L}: \text { Film reflectivity }\end{array}$ \\
\hline $\begin{array}{l}\text { Gas space (no optical } \\
\text { function) }\end{array}$ & - & $\left(\begin{array}{ll}0 & 1 \\
1 & 0\end{array}\right)$ & $M_{-}=U$ \\
\hline
\end{tabular}

The experimental characterization of the magnitudes included in transfer matrices of Table 1 is obtained from spectrophotometric measurements of the basic configurations listed in Table 2. Section 4 includes the procedure for the deduction of these equations, which can be systematically obtained by inverting relation (2). The expressions for the most complex cases, identified in Table 2, will be derived. The remaining formulae may be constructed analogously or through equivalent procedures from the literature. 
Table 2: Basic configurations for the experimental characterization of components.

\begin{tabular}{|c|c|c|c|}
\hline Configuration & Symbol & Magnitudes & References \\
\hline $\begin{array}{l}\text { Monolithic glass } \\
\text { (uncoated) }\end{array}$ & [S] & $\begin{array}{l}r_{s}=\frac{\beta-\sqrt{\beta^{2}-4(2-R) R}}{2(2-R)} \\
\tau_{s}=\frac{R-r_{s}}{r_{s} T}=\frac{-\left(1-r_{s}\right)^{2}+\sqrt{\left(1-r_{s}\right)^{4}+4 r_{s}^{2} T^{2}}}{2 r_{s}^{2} T} \\
\beta=T^{2}-R^{2}+2 R+1\end{array}$ & $\begin{array}{l}\text { Furler (1991) } \\
\text { Rubin et al. }(1998) \\
\text { EN } 410(1998,2011)\end{array}$ \\
\hline $\begin{array}{l}\text { Laminated glass } \\
\text { (no internal reflectivity) }\end{array}$ & {$\left[S_{1}\right]+\left[S_{2}\right]$} & $\tau_{L}^{*}:$ Formula (12) & $\begin{array}{l}\text { Rubin et al. }(1998)\left(\mathrm{S}_{1}=\mathrm{S}_{2}\right) \\
\text { EN } 410(1998) \\
\text { This paper }\end{array}$ \\
\hline $\begin{array}{l}\text { Laminated glass } \\
\text { (internal reflectivity) }\end{array}$ & {$[S)+(S]$} & $\tau_{L}, \rho_{L}:$ Formulas (14) & This paper \\
\hline $\begin{array}{l}\text { Coated monolithic glass } \\
\text { (external coating) }\end{array}$ & [S] & $\begin{array}{l}r_{o f}=\frac{R_{f}-r_{s}}{\left[1+r_{s}\left(R_{f}-2\right)\right] \tau_{s}^{2}} \\
t_{0}=\frac{T\left(1-r_{s} r_{o f} \tau_{s}^{2}\right)}{\left(1-r_{s}\right) \tau_{s}} \\
r_{o b}=R_{b}-\frac{t_{0}^{2} r_{s} \tau_{s}^{2}}{1-r_{s} r_{o f} \tau_{s}^{2}}\end{array}$ & $\begin{array}{l}\text { Rubin et al. }(1998) \\
\text { EN } 410(1998,2011) \\
\text { Baenas \& Machado (2009) }\end{array}$ \\
\hline $\begin{array}{l}\text { Coated laminated glass } \\
\text { (embedded coating) }\end{array}$ & {$\left[S_{1}\right]+\left[\left(S_{2}\right]\right.$} & $t_{0}, r_{o f}, r_{o b}:$ Formulas (11) & $\begin{array}{l}\text { Baenas \& Machado (2009) } \\
\text { EN } 410 \text { (2011) } \\
\text { This paper (including } \\
\text { singular solutions) }\end{array}$ \\
\hline
\end{tabular}

The total transmittance of a glazing system separating two optically identical media (air) is symmetrical for front and back incidence. This implies that the transmissivity of every optical interface in the system is also symmetrical, which can be demonstrated within the optical model ${ }^{\dagger}$, by taking determinants in the matrix product (2) and considering that $\operatorname{det}\left(M_{i}\right)=-\tau_{i b} / \tau_{i f}$ and $\operatorname{det}\left(M_{1, n}\right)=-1$ for the symmetrical system.

The study of some of the intrinsic properties of the model and their extension to other type of results (e.g. Section 6) will require the recovery of the basis of the model, i.e., the optical interfaces and their transfer matrices (1).

\section{GENERAL SOLUTION FOR LAMINATED GLASS WITH INTERNAL AND EXTERNAL COATINGS}

A glazing system with " $\mid\left[\mathrm{S}_{1}\right]+\left[\left(\mathrm{S}_{2}\right] \mid\right.$ " structure will be considered in order to model the optical performance of a laminated glass which may include an internal coating usually referred to as embedded coating - and two external coatings. The corresponding equation system is obtained from expressions (2), (3) and Table 1, from which the transfer matrix of the system is given by,

$$
M_{1,6}=M_{\mid[, 1} M_{S_{1,2}} M_{]+[, 3} M_{(, 4} M_{S_{2}, 5} M_{] \mid, 6}
$$

And therefore the energy coefficients of the glazing system, from (4) can be written as,

\footnotetext{
† It can be noted that this result is also obtained within the characteristic matrix theory, as shown by Abelès (1950), Theorem I: "The transmission factor of any stratified medium (absorbing or not) is independent of the sense of wave propagation". The proof of this theorem is made by induction, by showing that the product of $n$ characteristic matrices, ordered from front to back and vice versa, always has the same diagonal elements. Symmetry is fulfilled by energy factors, not by Fresnel coefficients relating the electromagnetic field components.
} 


$$
\begin{gathered}
T=\frac{t_{1} t_{2} t_{0} \tau_{1} \tau_{2} \tau_{L}}{\Delta}, \\
R_{f}=r_{1 f}+\frac{\left(1-r_{0 b} r_{2 f} \tau_{2}^{2}\right) r_{0 f} t_{1}^{2} \tau_{1}^{2} \tau_{L}^{2}+r_{2 f} t_{1}^{2} t_{0}^{2} \tau_{1}^{2} \tau_{2}^{2} \tau_{L}^{2}}{\Delta}, \\
R_{b}=r_{2 b}+\frac{\left(1-r_{1 b} r_{0 f} \tau_{1}^{2} \tau_{L}^{2}\right) r_{0 b} t_{2}^{2} \tau_{2}^{2}+r_{1 b} t_{2}^{2} t_{0}^{2} \tau_{1}^{2} \tau_{2}^{2} \tau_{L}^{2}}{\Delta},
\end{gathered}
$$

where $\Delta$ has been defined for convenience as

$$
\Delta=\left(1-r_{1 b} r_{0 f} \tau_{1}^{2} \tau_{L}^{2}\right)\left(1-r_{2 f} r_{0 b} \tau_{2}^{2}\right)-r_{2 f} r_{1 b} t_{0}^{2} \tau_{1}^{2} \tau_{2}^{2} \tau_{L}^{2}
$$

In (6), transmissivity and reflectivities for each component (see Fig. 1) are as follows: $\left(t_{1}, r_{1 f}, r_{1 b}\right)$ for the front coating " $\mid\left[{ }^{\prime \prime},\left(\tau_{1}, 0,0\right)\right.$ for the glass substrate "S $\mathrm{S}_{1}$ ", $\left(\tau_{L}, 0,0\right)$ for the polymer film " $]+\left[{ }^{\prime \prime},\left(t_{0}, r_{0 f}, r_{0 b}\right)\right.$ for the internal film-glass coating " (",,$\left(\tau_{2}, 0,0\right)$ for the glass substrate " $\mathrm{S}_{2}$ ", $\mathrm{y}\left(t_{2}, r_{2 f}, r_{2 b}\right)$ for the back coating "]|". The experimental characterization of these magnitudes is performed according to Table 2.

It should be noted that the first equation in (6), corresponding to glazing transmittance, can be written as $T=\tau_{m} / \Delta$, where $\tau_{m}$ represents the minimum transmissivity of the system (the product of the transmissivities of all the components). The $\Delta^{-1}>1$ factor accounts for the contribution of the structure of the system to the energy transmission (due to internal reflections). This formal relation applies to any glazing system.

The general solution to (6) in variables $\left(t_{0}, r_{0 f}, r_{0 b}\right)$ (i.e., the experimental characterization of the internal coating) may be obtained either by directly solving the corresponding equations or from matrix relation (5), which allows obtaining the transfer matrix for the internal coating, in the case of non-zero transmissivities:

$$
M_{(, 4}=\left(M_{\mid[, 1} M_{S_{1,2}} M_{]+[, 3}\right)^{-1} M_{1,6}\left(M_{S_{2}, 5} M_{] \mid, 6}\right)^{-1} .
$$

Relations like (7) provide a systematic procedure for the characterization of any glazing component. In the case under consideration,

$$
\begin{gathered}
t_{0}=\frac{1}{\tilde{\Delta}} \frac{t_{1} t_{2} T}{\tau_{1} \tau_{2} \tau_{L}} \\
r_{o f}=\frac{1}{\tilde{\Delta}} \frac{\left(R_{f}-r_{1 f}\right)\left(t_{2}^{2}-r_{2 f} r_{2 b}\right)-r_{2 f}\left(T^{2}-R_{f} R_{b}\right)-r_{1 f} r_{2 f} R_{b}}{\tau_{1}^{2} \tau_{L}^{2}} \\
r_{o b}=\frac{1}{\tilde{\Delta}} \frac{\left(t_{1}^{2}-r_{1 f} r_{1 b}\right)\left(R_{b}-r_{2 b}\right)-r_{1 b}\left(T^{2}-R_{f} R_{b}\right)-r_{1 b} r_{2 b} R_{f}}{\tau_{2}^{2}}
\end{gathered}
$$

where

$$
\begin{gathered}
\tilde{\Delta}=R_{f} r_{1 b}\left(t_{2}^{2}-r_{2 f} r_{2 b}\right)+R_{b} r_{2 f}\left(t_{1}^{2}-r_{1 f} r_{1 b}\right)-r_{1 b} r_{2 f}\left(T^{2}-R_{f} R_{b}\right) \\
+\left(t_{1}^{2}-r_{1 f} r_{1 b}\right)\left(t_{2}^{2}-r_{2 f} r_{2 b}\right) .
\end{gathered}
$$


Equations (6) and (8) are in accordance, except for notation issues, with EN standard 410:2011, annex B. In this standard, the corresponding expressions are obtained through the equivalent method of recurrence relations derived by Rubin (1982). Equivalent expressions to (6) were also used by Marco et al. (2001), although in that work the coating characterization was performed by numerical optimization, as a multilayer coating with known thicknesses and refractive indexes.

\subsection{SINGULAR SOLUTIONS AND GLAZING SYSTEMS WITH LOW OR NULL TRANSMITTANCE}

Solutions of the type (8) are sensitive to small denominators due to low transmissivities of some components, for specific regions of the solar radiation spectrum. A numerical compensation is often found when the transmission and reflection spectra of the whole glazing are recomposed. This compensation tends to cover the numerical problem in the components characterization, but may however imply a loss of accuracy when the characterization of the low transmissivity component is used in a different configuration. The factors taking part in this problem are the experimental accuracy in spectrophometric measurements, the theoretical approximations made in the modeling and the limitations in the manufacturing processes of the glazing systems used as basic configurations (Baenas and Machado 2009). A related problem, from a formal perspective, is the characterization of components in opaque systems (with some of the transmissivities being exactly zero), as is the case, e.g., for laminated crystalline silicon photovoltaic modules. Therefore, it is convenient to provide the singular solutions of the equation system (6), associated to the presence of null transmissivities. These singular solutions identify both limit solutions and those which are mathematically undefined.

The complementary singular solutions to (9) are compiled in Table 3. The undefined character of some of the variables has a strictly mathematical origin associated to the procedure for components characterization from spectrophotometric measurements of the glazing system. Section 6.3 presents an example of application of these singular solutions. Cases with $t_{1}=0$ or $t_{2}=0$ have no solutions.

Table 3: Singular solutions for an embedded coating in laminated glass.

\begin{tabular}{|ll|}
\hline Case & Singular solutions \\
& $t_{0}, r_{0 f}:$ no solutions \\
$\tau_{1}=0, \tau_{L}=0$ & $r_{0 b}=\frac{R_{b}-r_{2 b}}{\left(t_{2}^{2}+r_{2 f} R_{b}-r_{2 f} r_{2 b}\right) \tau_{2}^{2}}$ \\
& $t_{0}, r_{0 b}:$ no solutions \\
& $r_{0 f}=\frac{R_{f}-r_{1 f}}{\left(t_{1}^{2}+r_{1 b} R_{f}-r_{1 f} r_{1 b}\right) \tau_{1}^{2} \tau_{L}^{2}}$ \\
\end{tabular}




\subsection{CHARACTERIZATION OF THE TRANSMISSIVITY OF POLYMER FILMS.}

Polymer films commonly used for laminated glass manufacturing, typically PVB polyvinyl butyral- and EVA -ethyl vinyl acetate- have a refractive index approximately equal to that of the glass substrate, within the wavelength range of solar radiation (Rubin et al. 1998). A null reflectivity of the glass-film interface may then be assumed to a good approximation. The characterization of this component, "]+[" (a film with no reflectivity) can be done in terms of its transmissivity $\tau_{L}^{*}$, from " $\left[\mathrm{S}_{1}\right]+\left[\mathrm{S}_{2}\right]$ " (Table 2 ) basic configuration. The equation system for this glazing is obtained from the corresponding matrix product (2) or from (6), by substituting $t_{i} \rightarrow 1-r_{s_{i}}$ and $r_{i f}, r_{i b} \rightarrow$ $r_{s_{i}}$, where $r_{s_{i}}$ is the reflectivity of the uncoated monolithic glass substrate $S_{i}$. For nonzero transmissivities $\left(\tau_{i} \neq 0\right)$, the expression for $\tau_{L}^{*}$ obtained from the first equation in (9) is,

$$
\tau_{L}^{*}=\frac{1}{\tau_{1} \tau_{2}} \frac{-\left(1-r_{s_{1}}\right)\left(1-r_{s_{2}}\right)+\sqrt{4 r_{s_{1}} r_{s_{2}} T^{2}+\left(1-r_{s_{1}}\right)^{2}\left(1-r_{s_{2}}\right)^{2}}}{2 r_{s_{1}} r_{s_{2}} T} .
$$

The asterisk in $\tau_{L}^{*}$ refers to the transmissivity of the component under the hypothesis of null reflectivity. Equation (9) generalizes the expression given by Rubin et al. (1998) for the particular case where $r_{s_{1}}=r_{s_{2}}$ (symmetric laminate), and it is in accordance with the expression given by EN 410:2011, annex B. In this case, the standard solves this problem from the solution for a single substrate system, "|[S]|", with equivalent transmissivity $\tau_{1} \tau_{L} \tau_{2}$ (this kind of equivalences is described in Baenas and Machado 2009). It is common to opt for a characterization of the (9) type, given that reflectance spectrophotometry usually involves a loss in accuracy, even if the three (6) equations are solvable in order to obtain $\tau_{L}^{*}$.

The transmissivity of a polymer film within an opaque optical system is either not defined or null. In this case, the system is equivalent to the trivial case $T=0, R_{f}=r_{s_{1}}$ and $R_{b}=r_{S_{2}}$, where (12) is not defined and (13) expressions are zero, as this is the only solution which is compatible with $T=0, \tau_{1} \neq 0$ and $\tau_{2} \neq 0$ in the "[ $\left.\mathrm{S}_{1}\right]+\left[\mathrm{S}_{2}\right]$ " system. For the same reason, there are no singular solutions in the case of null transmissivities of the glass substrates.

There are several different mechanisms to introduce reflectivity within a laminated glass, such as the presence of thin films (section 3), embedded or encapsulated components (as is the case for photovoltaic modules, addressed in section 5.2), or the interfaces between different refractive index media. Therefore, it is convenient to provide a component which models the case of an adhesive film including reflectivity in the interface with the glass substrate. The characterization of such a component will allow evaluating the accuracy of the null reflectivity hypothesis, which considerably simplifies the modeling of the system.

A symmetrical system of the type "[S)+(S]" will be thus considered, where ")+(" represents the "film with reflectivity" component. Due to symmetry issues, external and internal reflectances of the glazing system are equal, $R_{f}=R_{b} \equiv R$, and so are the transmissivities and reflectivities of the glass substrates, $r_{s_{1}}=r_{s_{2}} \equiv r_{s}, \tau_{s_{1}}=\tau_{s_{2}} \equiv \tau_{s}$. The transfer matrix of the glazing system will be given by (2), that is, $M_{1,5}=$ $M_{[, 1} M_{S, 2} M_{)+(, 3} M_{S, 4} M_{], 5}$. Given that the matrix structure of the component is $M_{)+(, 3}=$ 
$U M_{3} U$, where 3 is an optical interface with transmissivity $\tau_{L}$ and reflectivity $\rho_{L}$, the component can be characterized from,

$$
M_{)+(, 3}=\frac{1}{\tau_{L}}\left(\begin{array}{cc}
\rho_{L} & \tau_{L}^{2}-\rho_{L}^{2} \\
1 & -\rho_{L}
\end{array}\right)=\left(M_{[, 1} M_{S, 2}\right)^{-1} M_{1,5}\left(M_{S, 4} M_{], 5}\right)^{-1}
$$

By evaluating this expression and identifying matrix elements, the following expressions are obtained:

$$
\tau_{L}=\frac{1}{\tilde{\Delta}} \frac{\left(1-r_{S}\right)^{2} T}{\tau_{S}^{2}}, \quad \rho_{L}=\frac{1}{\tilde{\Delta}} \frac{R-r_{S}\left(T^{2}-R^{2}+1+2 R\right)+r_{s}^{2}(2-R)}{\tau_{s}^{2}},
$$

$\tilde{\Delta}$, defined in (11), is in this case:

$$
\tilde{\Delta}=1+2 r_{s}(R-2)-r_{s}^{2}\left(T^{2}-R^{2}+4 R-4\right) .
$$

In addition, it may be convenient to determine the value of the glass-film interface reflectivity, as well as the internal transmissivity of the ")+(" component. In order to do this, its structure in optical interfaces from the previous matrix $M_{3}$ is explicited as $M_{)+(, 3}=U M_{3} U=U M_{3 a} U M_{3 b} U M_{3 c} U$. Here, $3 \mathrm{a}$ and $3 \mathrm{~b}$ are simple interfaces with reflectivity $r_{L}$ and transmissivity $1-r_{L}$, and $3 c$ is an attenuation interface $t_{L}$. By identifying the result of this product with (13) the following equation system is obtained,

$$
\tau_{L}=\frac{t_{L}\left(1-r_{L}\right)^{2}}{1-r_{L}^{2} t_{L}^{2}}, \quad \rho_{L}=r_{L}+\frac{\left(1-r_{L}\right)^{2} r_{L} \tau_{L}^{2}}{1-r_{L}^{2} t_{L}^{2}}
$$

As could be expected, such equations correspond to a monolithic substrate type system (Furler 1991), as they describe the film with planoparallel interfaces embedded in a glass substrate (see Fig. 2). In this configuration, $\tau_{L}$ and $\rho_{L}$ play the role of transmittance and reflectance of the film system. The solutions to (12) are:

$$
\begin{gathered}
r_{L}=\frac{\beta-\sqrt{\beta^{2}-4\left(2-\rho_{L}\right) \rho_{L}}}{2\left(2-\rho_{L}\right)}, \quad \beta=\tau_{L}^{2}-\rho_{L}^{2}+2 \rho_{L}+1, \\
t_{L}=\frac{\rho_{L}-r_{L}}{r_{L} \tau_{L}}=\frac{-\left(1-r_{L}\right)^{2}+\sqrt{\left(1-r_{L}\right)^{4}+4 r_{L}^{2} \tau_{L}^{2}}}{2 r_{L}^{2} \tau_{L}}
\end{gathered}
$$

These solutions are formally equivalent to those of an "[S]" system (see Table 2). 


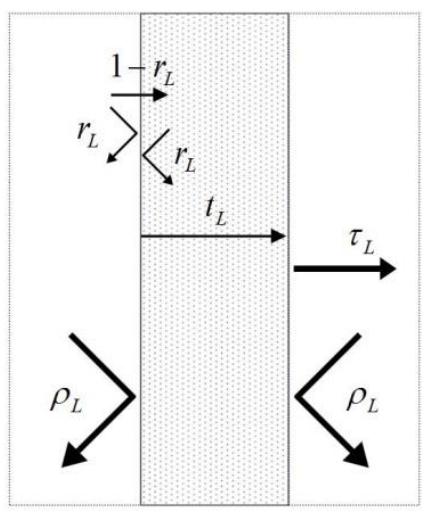

Fig. 2: Schematics of a film component with reflectivity.

The hypothesis of zero reflectivity in the glass-film interface means that $r_{L}=0$. In this case, from (12), $\rho_{L}=0$ and $\tau_{L} \equiv \tau_{L}^{*}=t_{L}$ is obtained. In any case, (11) is a characterization of the film transmissivity, dependent on the glazing $T$ and $R$ measurements.

Fig. 3 shows the values of $\tau_{L}^{*}(9)$ and $\tau_{L}(11)$, calculated transmissivity with and without null reflectivity hypothesis, for polymer films with different colors, in $4+4.1$ glass configurations (two clear float $4 \mathrm{~mm}$ nominal thickness glass and an intermediate 0.4 $\mathrm{mm}$ polymer film), taken from the same experimental characterization. It has been verified for all cases that the design hypothesis of zero reflectivity is acceptable. In the less favorable case (white PVB), reflectivity reaches a spectral maximum value of 0.06 in the visible spectrum range. For the rest of films and spectral ranges, reflectivity values are in the $10^{-2}$ order or lower. The average refractive index declared by manufacturers is 1.48 for the films and 1.52 for glass substrates (in accordance with, e.g., Rubin 1984). However, (11) characterization does not require the introduction within the formalism of refractive indexes through Fresnel equations, in order to justify the ad hoc hypothesis of null reflectivity for the modeling process. 


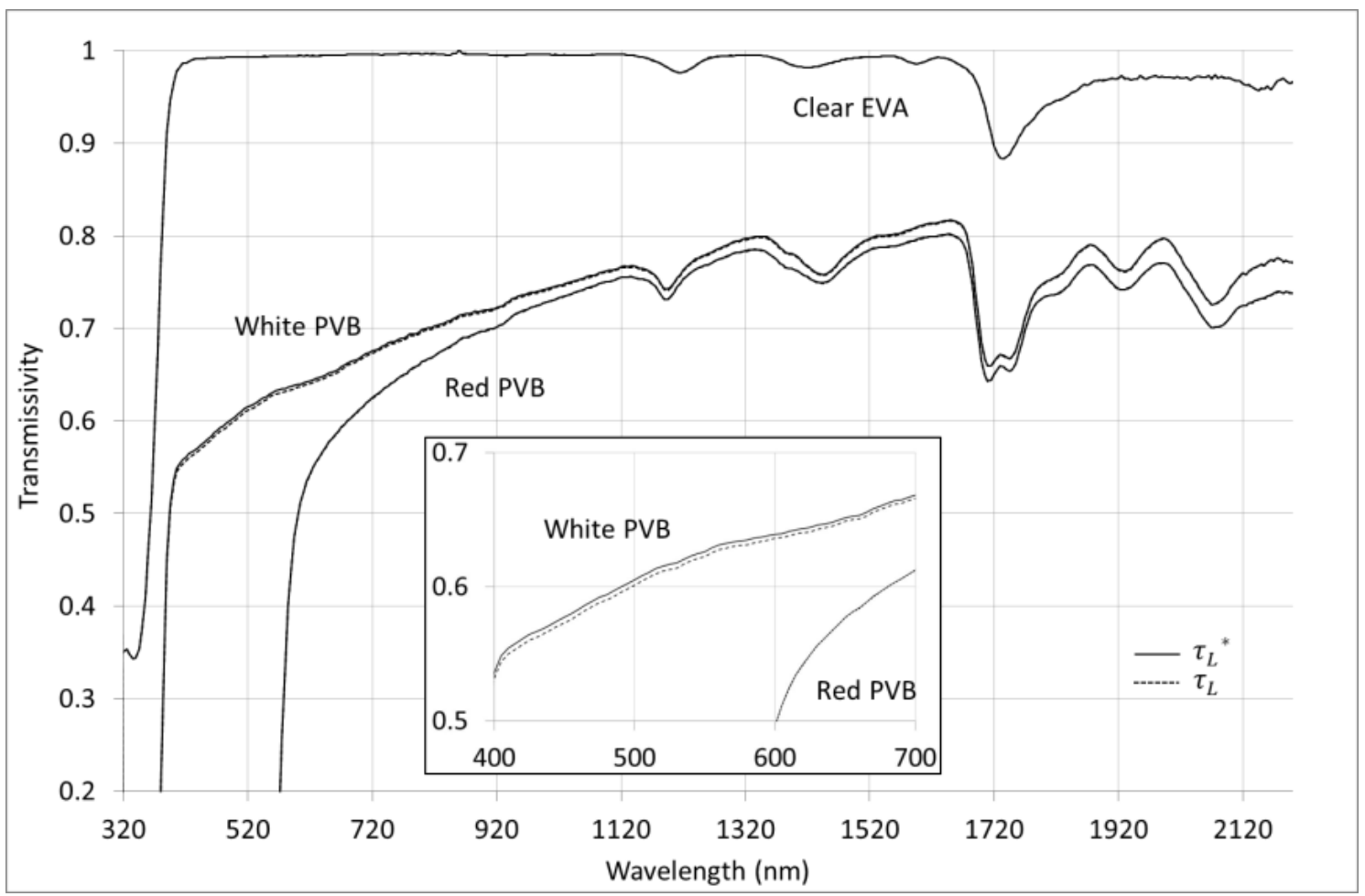

Fig. 3: Spectral transmissivity of polymer films, calculated with and without null reflectivity hypothesis.

\section{CALCULATION OF LAYER-BY-LAYER ABSORPTION}

Thermal studies of multilayer glazing systems formed by laminated glass generally adopt the simplification of considering the energy absorption for the whole system. This simplification is perfectly valid for the thermal balance of the system when it is obtained from the thermal resistances (in series association) of the laminated glass components and the short wavelength absorption is taken as $A=1-T-R_{f}$. However, glass prescriptions in terms of mechanical durability (admissibility of thermal tensions, or, equivalently breakage risk assessment) involve industrial processes of different manufacturing times and production costs (glass edge processing, thermal toughening or strengthening, mainly). It is therefore convenient to have a procedure to select, from the glazing design phase, the distribution of the energy absorptance of each laminated glass component, and thus their particular thermal breakage risk.

From the perspective of the physical modeling of the system, the evaluation of individual breakage risks requires the calculation of incoming $(I)$ and outgoing $(J)$ fluxes of each optical interface. This is done from relation (2) and the explicit evaluation of the intermediate fluxes or, equivalently, of the matrix product blocks corresponding to the real components of the system. From the knowledge of the fluxes at both sides of a i-th optical interface, absorptivity is given by the incoming and outgoing energy balance, assuming unitary solar irradiance:

$$
A_{i}=I_{i f}-J_{i f}+I_{i b}-J_{i b}
$$

An iterative procedure is thus defined (see e.g. ISO 15099 standard, Maestre 2000 or Van Nijnatten 1994), which does not directly provide closed analytical expressions for 
the energy fluxes and therefore is solved numerically. However, it will be proved in section 5 that it is possible to obtain analytical expressions for the layer-by-layer absorption of a laminated glass from the recurrence relations between the energy flux vectors and a convenient manipulation of the transfer matrices associated to the optical components. These expressions are not, to our best knowledge, included in any previous reference and, however, have a similar applicability as those for transmissivity and reflectivity provided by standards.

In order to lighten notation, flux vectors will be written as $\left(I_{f / b}, J_{f / b}\right)^{t}=\Psi_{f / b}$, so that given the $\mathrm{i}$-th interface with transfer matrix $M_{i}, \Psi_{i, f}=M_{i} \Psi_{i, b}$ is obtained. By considering the definition of the link matrix (1), it is found that $\Psi_{i, b}=U \Psi_{i+1, f}$ and $\Psi_{i, f}=U \Psi_{i-1, b}$. These relations are equivalent, given the fact that $U^{-1}=U$. By relating internal and external fluxes through $M_{i}$, the following recurrence relations are obtained, which are equivalent and independent for the fluxes at each interface,

$$
\Psi_{i, f}=M_{i} U \Psi_{i+1, f}, \quad \Psi_{i, b}=U M_{i+1} \Psi_{i+1, b}, \quad(i=1,2, \ldots, n-1)
$$

These expressions allow an iterative solution of the calculation from the irradiance and radiosity values of the whole optical system, given by (2).

\section{EXAMPLES OF APPLICATION}

\subsection{LAYER-BY-LAYER ABSORPTION IN A CONVENTIONAL LAMINATED GLASS}

As an example of calculation of analytical expressions for the absorption of components of a laminated glass (that is, in terms of transmissivities and reflectivities of the optical components), the case of a " $\left|\left[S_{1}\right]+\left[S_{2}\right]\right|$ " system will be studied here. The system is a conventional laminated glass with two coated glass panes (external coating) and a polymer film with no reflectivity in the glass-film interface. Within the application of the optical method presented herein, the system is formed by 5 optical components. However, in order to simplify the iterative procedure and obtain simpler analytical expressions, the number of optical components may be reduced to 3 for the calculation of fluxes and absorptivities, by using the following combined components: "| $\left[\mathrm{S}_{1}\right.$ " (external glass), "]+[" (polymer film) y " $\left.\mathrm{S}_{2}\right] \mid$ " (internal glass). The simplification does not hinder its practical applicability, given that only the energy absorptance of the real components is interesting, i.e., glass and polymer film, and not their division for the sake of modeling. In order to clarify notation, an asterisk will be used for transfer matrices associated to combined elements, $M_{1}^{*}, M_{L}^{*}$ and $M_{2}^{*}$ respectively for external glass, polymer film and internal glass. For the whole system the relation $\Psi_{1, f}=$ $M_{1,3} \Psi_{3, b}$ applies. From (3) expressions $\Psi_{1, f}$ and $\Psi_{3, b}$ can be calculated, i.e.,

$$
J_{3 b}=\frac{1}{m_{12}}, \quad J_{1 f}=\frac{m_{22}}{m_{12}}
$$

Where $m_{i j}$ are the elements of the system matrix, $M_{1,3}$. Applying recurrence relations (15), flux vectors at the interfaces between the combined components are obtained. In particular, $\Psi_{2, b}$ and $\Psi_{1, b}$ are calculated from the relation for internal fluxes $(b)$ : 


$$
\begin{gathered}
\left(\begin{array}{c}
I_{2 b} \\
J_{2 b}
\end{array}\right)=U M_{2}^{*}\left(\begin{array}{c}
0 \\
1 / m_{12}
\end{array}\right)=\left(\begin{array}{l}
m_{22}^{(2)} / m_{12} \\
m_{12}^{(2)} / m_{12}
\end{array}\right), \\
\left(\begin{array}{c}
I_{1 b} \\
I_{1 b}
\end{array}\right)=U M_{L}^{*}\left(\begin{array}{l}
m_{22}^{(2)} / m_{12} \\
m_{12}^{(2)} / m_{12}
\end{array}\right)=\left(\begin{array}{l}
m_{21}^{(L)} m_{22}^{(2)} / m_{12}+m_{22}^{(L)} m_{12}^{(2)} / m_{12} \\
m_{11}^{(L)} m_{22}^{(2)} / m_{12}+m_{12}^{(L)} m_{12}^{(2)} / m_{12}
\end{array}\right),
\end{gathered}
$$

where the superscript $(1,2$ o L) of the matrix elements refers to the original component to which the matrix relates (without superscript for $M_{1,3}$ ).

It should be noted that, in order to determine the analytical form of the absorptivities, transfer matrices $M^{*}$ do not coincide with those from Table 1, given that combined components are being used and the link matrix $U$ has been included within recurrence relations (18) instead of the optical component matrix. In terms of (1) matrices for the system interfaces, these are constructed as $M_{1}^{*}=M_{1} U M_{2}, M_{L}^{*}=M_{3}$ and $M_{2}^{*}=M_{4} U M_{5}$, where 1 and 5 are interfaces with transmissivity and reflectivity (coatings) and 2, 3 and 4 are interfaces with just transmissivity (glass substrates and polymer film). The corresponding explicit expressions are,

$$
\begin{gathered}
M_{1}^{*}=\frac{1}{t_{1}}\left(\begin{array}{cc}
-r_{1 b} \tau_{1} & \frac{1}{\tau_{1}} \\
\tau_{1}\left(t_{1}^{2}-r_{1 f} r_{1 b}\right) & \frac{r_{1 f}}{\tau_{1}}
\end{array}\right), \quad M_{2}^{*}=\frac{1}{t_{2}}\left(\begin{array}{cc}
-\frac{r_{2 b}}{\tau_{2}} & \frac{1}{\tau_{2}} \\
\tau_{2}\left(t_{2}^{2}-r_{2 f} r_{2 b}\right) & r_{2 f} \tau_{2}
\end{array}\right), \\
M_{L}^{*}=\left(\begin{array}{cc}
0 & \frac{1}{\tau_{L}} \\
\tau_{L} & 0
\end{array}\right) .
\end{gathered}
$$

From (14), (17) and (18) the absorptivities of the combined external glass $\left(A_{1}\right)$, polymer film $\left(A_{L}\right)$ and internal glass $\left(A_{2}\right)$ components are obtained:

$$
\begin{gathered}
A_{1}=\frac{1-r_{1 f}+t_{1} \tau_{1}\left(r_{2 f} \tau_{L}^{2} \tau_{2}^{2}-1\right)+r_{2 f} \tau_{1}^{2} \tau_{L}^{2} \tau_{2}^{2}\left(r_{1 f} r_{1 b}-r_{1 b}-t_{1}^{2}\right)}{1-r_{1 b} r_{2 f} \tau_{1}^{2} \tau_{L}^{2} \tau_{2}^{2}}, \\
A_{L}=\frac{t_{1} \tau_{1}\left(1-\tau_{L}\right)\left(1+r_{2 f} \tau_{L} \tau_{2}^{2}\right)}{1-r_{1 b} r_{2 f} \tau_{1}^{2} \tau_{L}^{2} \tau_{2}^{2}}, \\
A_{2}=\frac{t_{1} \tau_{1} \tau_{L}\left(1-t_{2} \tau_{2}-r_{2 f} \tau_{2}^{2}\right)}{1-r_{1 b} r_{2 f} \tau_{1}^{2} \tau_{L}^{2} \tau_{2}^{2}} .
\end{gathered}
$$

It can be proved that the absorption of the whole system, $A$, verifies,

$$
A=A_{1}+A_{L}+A_{2}=I_{1 f}-J_{1 f}+I_{3 f}-J_{3 f}=1-T-R_{f} .
$$

Note that the denominator in (20) coincides with that of (6), $\Delta$, if the internal coating is eliminated, i.e., with $t_{0}=1, r_{0 f}=r_{0 b}=0$.

As an example of numerical representation of the model results, Fig. 4 depicts the spectral absorptivity of the components of a symmetrical laminated glass $(4+4.1)$ formed by two $4 \mathrm{~mm}$ (nominal thickness) clear float glass panes and an internal 0.38 
mm PVB polymer film. Spectral absorptivity can be integrated (weighted average) following EN 410:2011 standard in order to obtain the energy absorption of each component for the attainment of thermal balances. The experimental measurements of transmittance and reflectances of monolithic panes (in isolated setting) and laminated glass (thick line in Fig. 4) have been performed with a Perkin Elmer Lambda 950 spectrophotometer equipped with a $150 \mathrm{~mm}$ integrating sphere.

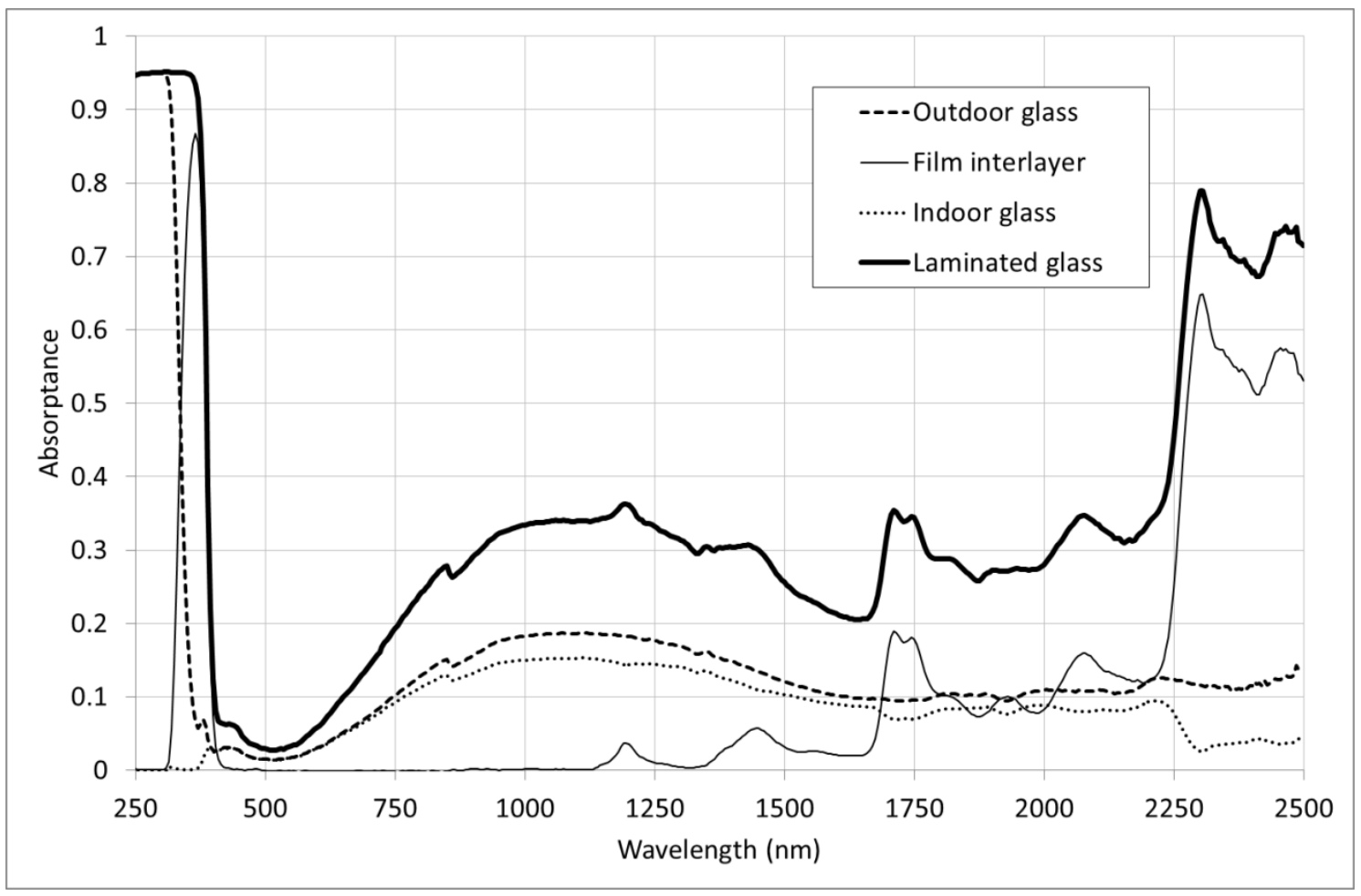

Fig. 4: Absorptivities of the components of a laminated glass.

To the best of our knowledge, there is no technology that allows a direct measurement of the layer-by-layer absorption within a glazing system. Related standards as ISO 15099 or EN 13363, rely on numerical procedures that have been accepted with a basis on their accordance with derived effects like the thermal balances (Finlayson et al. 1993, van Dijk 1966). In this sense, the content of the next section is also a derived effect, due to the relation between the internal absorption and the conversion efficiency of a photovoltaic module.

\subsection{MODELING OF A LAMINATED PHOTOVOLTAIC MODULE}

Photovoltaic (PV) modules encapsulated in laminated glass, with the PV cells embedded in the polymer film, are a particular case of glazing system with opaque regions. In this kind of systems, the interest of the modeling lies in the determination of the spectral absorptivity of the encapsulated cell, which is different from the measured absorptivity in air-cell configuration, due to the variation of reflectivity in the interface. This absorptivity is directly related to the cell efficiency. An accurate modeling of the energy absorptance of the PV cell should consider, when possible, the cell surface roughness and the consequent light dispersion at the interface, leading to equation systems which have to be solved numerically (see e.g. Santbergen and Zolingen 2008, 
based on the multidirectional net-radiation method). Herein, however, only planoparallel interfaces will be considered, in line with the modeling hypotheses of previous sections, which allows obtaining closed analytical expressions in the order of accuracy provided by the standards.

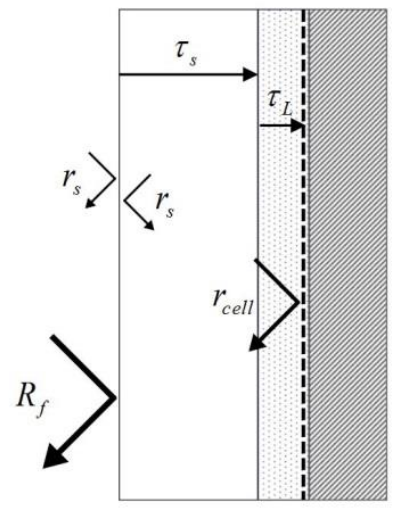

Fig. 5: Schematics of laminated glass-PV cell structure.

This glazing system (Fig. 3) may be studied from the singular solution to the equation system (6) given in Table 3 for the $\tau_{2}=0$ case, which determines the spectral reflectivity of the encapsulated PV cell, $r_{o f} \equiv r_{c e l l}$.

Given that there is no likelihood of confusion, $\tau_{1} \equiv \tau_{s}$ will be used. By restricting to the usual case of an uncoated external glass $\left(r_{1 f}=r_{1 b} \equiv r_{s}, t_{1}=1-r_{s}\right)$, the resulting expression is,

$$
r_{\text {cell }}=\frac{R_{f}-r_{S}}{\tau_{S}^{2} \tau_{L}^{2}\left(1-2 r_{S}+R_{f} r_{S}\right)}
$$

Symbolically the modeling of the system is " $\left[\mathrm{S}_{1}\right]+\left[\left(\mathrm{P}^{\prime}\right.\right.$, where " $\mathrm{P}$ " represents the opaque panel, which cancels the optical influence of any other components at its back side. Here the internal coating component "(" has a virtual character in the sense that it allows the introduction of reflectivity in the system $\left(r_{\text {cell }}\right)$ but it is not associated to the existence of a real thin film within the glazing system. It should be also noted that expressions (19) are not applicable in this case, given that they describe a system with zero internal reflectivity. Therefore, it is required to reconstruct the absorptivities by following a procedure analogous to section 5.1, from which only differences will be outlined herein, in order to lighten the exposition.

Starting from the " $\left[\mathrm{S}_{1}\right]+\left[\left(\mathrm{S}_{2}\right]\right.$ " system, the absorptances corresponding to the relevant combined components are obtained. These components are, in this case, $M_{1}^{*}=$ $M_{1} U M_{2} U M_{3}, M_{2}^{*}=U M_{4} U$ and $M_{3}^{*}=M_{5} U M_{6}$, where 1 and 6 are air-glass and glass-air interfaces, 4 is the interface corresponding to reflectivity $r_{\text {cell }}$ and 2,3 and 5 are interfaces with just transmissivity (substrate plus polymer film - joint for convenienceand opaque panel substrate). $M_{5}$ transmissivity is null $\left(\tau_{2}=0\right)$ and the internal interface $M_{6}$ does not affect the optical performance of the system. The absorptances for the combined components are denoted $A_{1}, A_{2}$ and $A_{3}$ respectively. As indicated in section 4.1, the singular solution for $\tau_{2}=0$ implies the indefinition of $t_{0}$ and $r_{o b}$, which, 
however, enter the analytical expression for the absorptivities. Nevertheless, this lack of definition affects only the characterization of the component "(" from the global coefficients of the glazing. In this new system, it can be considered, without a loss of generality, that the film-panel interface is a simple interface and therefore $t_{0}=1-r_{\text {cell }}$, $r_{o b}=r_{c e l l}$. Despite $r_{o b}$ does not seem to (directly) influence the system, it is not possible to cancel its value as this would imply a characterization of the related interface with no physical sense.

Under the above mentioned modeling conditions, the analytical expressions for the absorptivities of the whole system (photovoltaic module, $A_{\text {mod }}$ ), opaque panel (PV cell, $\left.A_{\text {cell }}\right)$ and the glass-encapsulation film $\left(A_{\text {enc }}\right)$, are given by,

$$
\begin{gathered}
A_{\text {mod }}=A_{1}+A_{2}+A_{3}=1-T-R_{f}=\left(1-r_{s}\right) \frac{1-r_{\text {cell }} \tau_{s}^{2} \tau_{L}^{2}}{1-r_{s} r_{\text {cell }} \tau_{s}^{2} \tau_{L}^{2}} \\
A_{\text {cell }}=A_{2}+A_{3}=\left(1-r_{s}\right)\left(1-r_{\text {cell }}\right) \frac{\tau_{s} \tau_{L}}{1-r_{s} r_{\text {cell }} \tau_{s}^{2} \tau_{L}^{2}} \\
A_{\text {enc }}=A_{1}=A_{\text {mod }}-A_{\text {cell }} .
\end{gathered}
$$

Fig. 6 shows the absorptivities of a 4+4.1 PV module (two clear float glass substrates, 4 $\mathrm{mm}$ nominal thickness plus a $0.4 \mathrm{~mm}$ EVA encapsulation film). The UV-Vis-NIR spectrophotometric measurements for the reflectance of the system $\left(R_{f}\right)$ and the characterization of the glass substrate and polymer film were performed with a JASCO V-670 spectrophotometer equipped with a $150 \mathrm{~mm}$ integrating sphere.

The figure shows the variation in absorptivity of the encapsulated cell with respect to measured bare cell absorptivity. The $A_{\text {cell }}$ value is related to the intensity of generated electric current, and therefore to cell efficiency. It also participates in the equations of the thermal balance of the module through Joule effect (Fung and Yang 2008). It can be proved (Machado et al. 2015, in preparation) that the method accurately matches short-circuit current density values of a PV module as obtained from experimental External Quantum Efficiency measurements. From a reduced set of experimental measurements of a reference encapsulation scheme, precise predictions of PV module efficiencies in different encapsulation conditions can thus be performed with the method presented herein. 


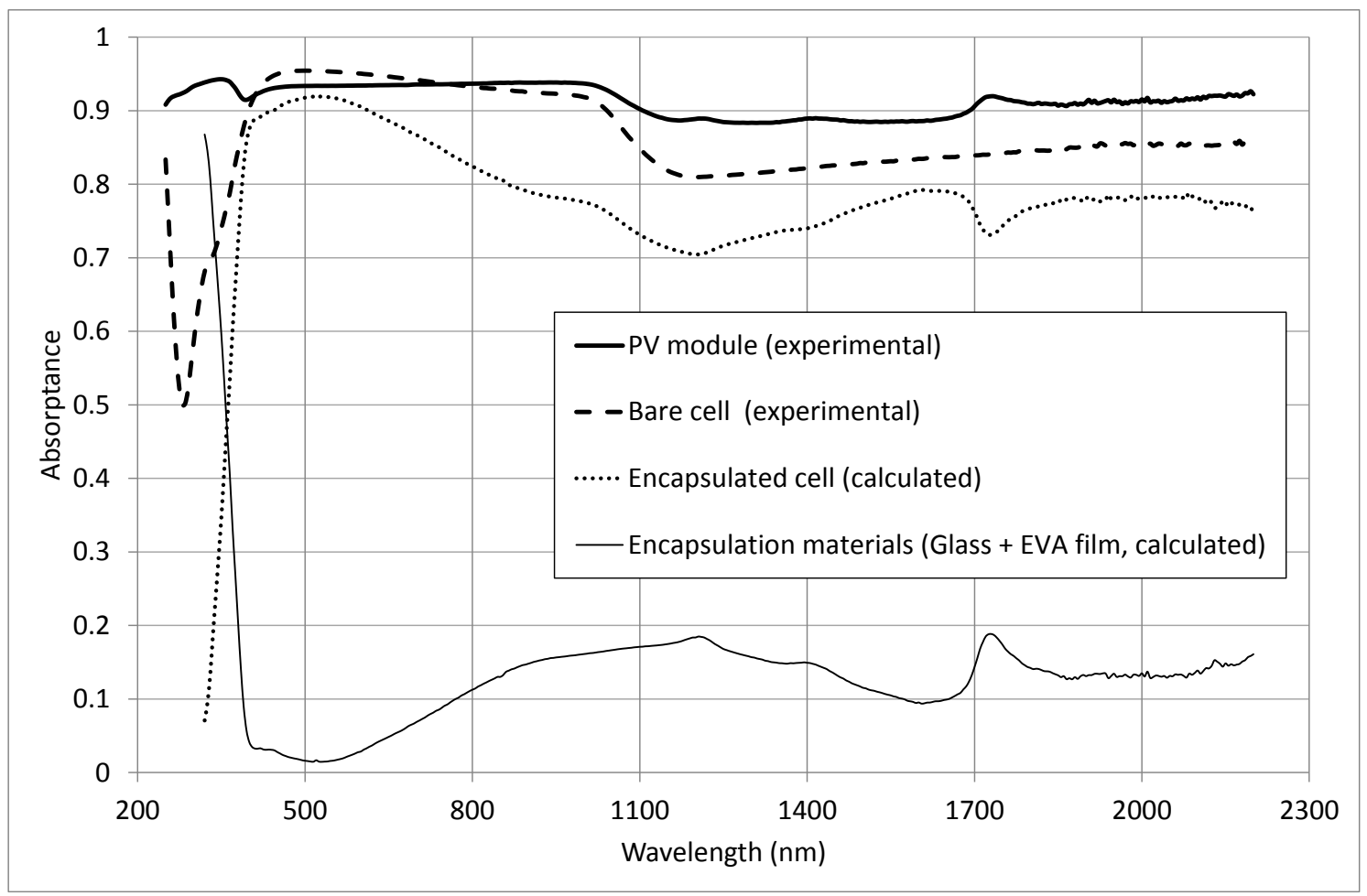

Fig. 6: Absorptivities in a PV module with front glass encapsulation

\section{CONCLUSIONS}

The optical model presented herein allows the optical and energy study of glazing systems under the modeling conditions of the current normative framework. Following the guideline for the proposal of closed analytical expressions for the magnitudes describing the short-wave energy performance of the system, the method is based on a convenient definition of optical components and their associated transfer matrices.

A systematic procedure has been established for the definition of the equations describing the glazing system from the ordered assembly of its components. The necessary expressions for the experimental characterization of the glazing components from the experimental measurements of specific configurations have also been obtained. It has been proved that transfer matrices associated to optical components and glazing system are symmetric in transmission by consistency of the model.

This methodology has been applied to a general laminated glass, with the possibility of including internal and external selective coatings. The study of such a system has focused on the characterization of the internal coatings, including the singular solutions for the case of opaque components, and the characterization of polymer film transmissivity. This characterization takes into account the reflectivity of the polymer film-glass interface, which has allowed proving the accuracy of the (normative) hypothesis about the null reflectivity of commonly used polymer films. Current normative references do not include this specific characterization, despite the fact that it requires the same kind of experimental measurements than the characterization of other components (glass or coatings), and has a wider accuracy and range of applicability. 
The recurrence relations allowing the determination of incoming and outgoing energy fluxes at the optical interfaces have been established. A systematic procedure providing closed analytical expressions for the absorptances of the components of a glazing system has been presented. This procedure is based on an appropriate definition of (combined) components, their associated transfer matrices, and the application of the general recurrence relations.

As an example of application, the analytical expressions for the absorptance of the components of a conventional laminated glass (glass-polymer film-glass with external selective coatings) have been presented. Although these expressions are not included in current standards, they are highly useful during the design phase of a laminated glass, as they provide a means to determine differentiated absorption at each glass pane, and therefore, their individual thermal breakage risk. Additionally, the computational cost of the expressions provided in this work is analogous to the expressions included in the standards for the characterization of components or the calculation of system transmittance and reflectance.

The expressions for the layer-by-layer absorptance of a photovoltaic module with a glass superstrate and cells embedded in a polymer film have also been provided. The opaque parts (PV cells) require the use of the singular solutions, previously derived, in order to get the cell reflectivity. This allows establishing a comparison between the absorptivity of the encapsulated cell and the one obtained from an air-bare cell measurement. The absorptivity of the cell in encapsulated conditions may be used for efficiency calculations of PV modules in different encapsulation schemes.

\section{ACKNOWLEDGMENTS}

M.M. acknowledges partial support from KIC InnoEnergy through BIPV INSIGHT project.

\section{REFERENCES}

[1] Abelès, F., 1950. Recherches sur la propagation des ondes électromagnétiques sinusoidales dans les milieux stratifiés. Application aux couches minces. Annales de Physique 5, 596-640, 706-782.

[2] Baenas, T., Machado, M., 2009. Optical simulation of laminated glass. Proc. Glass Performance Days 2009. Ed. GPD-Glaston Finland Oy, 742-745.

[3] Born, M., Wolf, E., 1959. Principles of optics. First ed. Pergamon Press.

[4] Centurioni, E., 2005. Generalized matrix method for calculation of internal light energy flux in mixed coherent and incoherent multilayers. Applied Optics, 44 (35), 7532-7539.

[5] Epstein, L.I., 1952. The Design of Optical Filters. J. Opt. Soc. Am., 42 (11), 806-810.

[6] EN 13363-1:2003 (European Standard). Solar protection devices combined with glazing. Calculation of solar and light transmittance. Simplified method.

[7] EN 410:2011 (European Standard). Glass in building. Determination of luminous and solar characteristics of glazing.

[8] EN 673:2011 (European Standard). Glass in building. Determination of thermal transmittance (U value). Calculation method.

[9] Finlayson, E.U., Arasteh D.K., Huizenga, C., Rubin M.D., and Reilly M.S, 1993. Window 4.0: Documentation of calculation procedures. Lawrence Berkeley Laboratory. University of California. 
[10] Francombe, M.H., Hoffman, R.W., 1971. Physics of Thin Films. Academic Press, Inc., New York.

[11] Fung, T.Y.Y., Yang, H., 2008. Study on thermal performance of semi-transparent building-integrated photovoltaic glazings. Energy and Buildings, 40, 341-350; doi: 10.1016/j.enbuild.2007.03.002.

[12] Furler, R.A., 1991. Angular Dependence of Optical Properties of Homogeneous Glasses. ASHRAE Transactions 1991, 97 (2).

[13] Harbecke, B., 1986. Coherent and incoherent reflection and transmission of multilayer structures. Appl. Phys. B 39, 165-170.

[14] Heavens, O.S., 1960. Optical properties of thin films. Rep. Prog. Phys, 23, 66-69; doi:10.1088/0034-4885/23/1/301.

[15] Herpin, A., 1947. Calcul du pouvoir reflecteur d'un système stratifié quelconque. Compt. rend., 225, 182-183.

[16] ISO 9050:2003 (International Standard). Determination of light transmittance, solar direct transmittance, total solar energy transmittance, ultraviolet transmittance and related glazing factors.

[17] ISO 15099:2003 (International Standard). Thermal performance of windows, doors and shading devices. Detailed calculations.

[18] Katsidis, C.C., Siapkas, D.I., 2002. General transfer-matrix method for optical multilayer systems with coherent, partially coherent, and incoherent interference. Applied Optics, 41 (19), 3978-3987.

[19] Maestre, I.R., 2000. Modelo óptico y térmico de acristalamientos complejos. Phd. Dissertation, University of Sevilla.

[20] Maestre, I.R., Molina, J.L., Ross, A., Coronel, J.F., 2006. A single-thin-film model for the angle dependent optical properties of coated glazings. Solar Energy, 81 (8), 969-976; doi: 10.1016/j.solener.2006.12.002.

[21] Marco, J.M., Uliaque, L., Villuendas, F., 2001. Diseño y producción de recubrimientos de control energético para su utilización en vidrio laminado. Bol. Soc. Esp. Cerám. Vidrio, 40 (2), 113-119.

[22] Mitsas, C.L., Siapkas, D.I., 1995. Generalized matrix method for analysis of coherent and incoherent reflectance and transmittance of multilayer structures with rough surfaces, interfaces, and finite substrates. Applied Optics, 34 (10), 1678-1683.

[23] Pfrommer, P., Lomas, K.J., Seale, C., Kupke, Chr., 1995. The radiation transfer through coated and tinted glazing. Solar Energy, 54 (5), 287-299; doi: 10.1016/0038092X(94)00132-W.

[24] Rubin, M., 1982. Solar optical properties of windows. Energy Research, 6, 123-133.

[25] Rubin, M., Rottkay, K.V., Powles, R., 1998. Window Optics. Solar Energy, 62 (3), 149-161; doi:10.1016/S0038-092X(98)00010-3.

[26] Santbergen, R., van Zolingen, R.J.C., 2008. The absorption factor of crystalline silicon PV cells: A numerical and experimental study. Solar Energy Materials and Solar Cells, 92, 432-444; doi:10.1016/j.solmat.2007.10.005.

[27] Thelen, A., 1989. Design of optical interference coatings. McGraw-Hill.

[28] van Dijk, H.A.L., 1996. WIS reference manual, TNO Building and Construction Research.

[29] Van Nijnatten, P.A., 1994. Mathematical modeling of optical glazing performance. Proc. SPIE 2255, Optical Materials Technology for Efficiency and Solar Energy Conversion XIII, 753; doi: 10.1117/12.185417.

[30] Van Nijnatten, P.A., 2001. A pseudo-Fresnel approach for predicting directional optical properties of coated glazing. Thin Solid Films, 392, 282-288; doi: 10.1016/S00406090(01)01044-6. 
[31] Machado, M., Baenas, T., Yurrita, N., 2016. Optical model for multilayer glazing systems: Experimental validation through the analytical prediction of encapsulationinduced variation of PV modules efficiency. Solar Energy, 135, 77-83;

doi: 10.1016/j.solener.2016.05.040 